\title{
LA CONDICIÓN INTERTEXTUAL EN LAS CITAS DE LOS ARTÍCUlOS DE INVESTIGACIÓN DE PSICOLOGÍA*
}

\author{
Juan Antonio González de Requena** \\ Carolina Andana*** \\ Claudio Duhart****
}

\section{Resumen}

Este artículo pretende poner de manifiesto las principales formas, funciones y tipos de fuente de la citación en dos revistas de Psicología chilenas. Se realizó un análisis de contenido de las citas de 106 artículos, publicados entre el 2008 y el 2012 en la Revista de Psicología y en la revista Psykhe. Los resultados muestran cierto predominio de prácticas de citación meramente referenciales, confirmatorias y bibliográficas, que podrían caracterizarse como metatextuales.

Palabras clave: Intertextualidad, citación, artículo de Investigación, Psicología.

\section{THE INTERTEXTUAL CONDITION IN QUOTES OF PSYCHOLOGY RESEARCH ARTICLES}

\begin{abstract}
This article aims to highlight the main forms, functions and types of source of citation in two Chilean journals of Psychology. A content analysis of citations of 106 articles, published between 2008 and 2012 in Revista de Psicología and Psykhe, was performed. The results show certain predominance of purely referential, confirmatory and bibliographic citation practices, which could be characterized as metatextual.
\end{abstract}

Keywords: Intertextuality, Citation, Research Paper, Psychology.

Recibido: 16-04-2014

Aceptado: 12-05-2015

* Artículo vinculado al proyecto Fondecyt de iniciación n 1121113, “La psicología como texto".

** Español. Doctor en Filosofía por la Universidad Complutense de Madrid. Académico de la Universidad Austral de Chile, Puerto Montt, Chile. jagref8@gmail.com

*** Chilena. Psicóloga por la Universidad Austral de Chile, Puerto Montt, Chile. carolina.andana@ gmail.com

**** Chileno. Psicólogo por la Universidad Austral de Chile, Puerto Montt, Chile. claudio.duhart@ gmail.com 


\section{Introducción}

Las citas desempeñan una función crucial en el discurso de la ciencia y, particularmente, en el género del artículo científico; a través de la citación, se conforman cuerpos compartidos de literatura disciplinar, extensas redes de comunicación científica y complejos vínculos de cooperación intelectual entre los investigadores. No es de extrañar que, en las últimas décadas, se haya desarrollado un creciente interés en investigar las prácticas de la citación, ya sea desde perspectivas funcionales que se preguntan por las diferentes razones pragmáticas para citar un texto (aportar evidencia, discutir o sostener una hipótesis, contextualizar históricamente un descubrimiento, etc.), ya sea desde perspectivas normativas que examinan los códigos disciplinares de citación y las reglas de atribución del crédito científico, o bien desde perspectivas sociales y psicológicas que consideran las motivaciones personales de los autores al citar a otros (Cronin, 1998). En ese sentido, cabe reconocer tres líneas principales de investigación interesadas en el análisis de las prácticas de citación: el enfoque bibliométrico que predomina en las Ciencias de la información; los estudios del discurso científico y de las formas de comunicación de la ciencia en Historia y Sociología de la ciencia, y, finalmente, la Lingüística aplicada, el análisis de los géneros del discurso científico y los estudios idiomáticos para propósitos académicos (White, 2004).

La bibliometría responde a la necesidad de indexación y recuperación de referencias bibliográficas, y aporta investigación cuantitativa sobre las frecuencias de citación y los índices de impacto de las publicaciones científicas. Progresivamente, los estudios bibliométricos de la citación han incorporado variables como el índice de impacto de los autores y las publicaciones, la vida media de las referencias citadas, y la densidad citacional o número de referencias citadas en cada fuente (Garfield, 2009). De ese modo, la investigación bibliométrica pretende explicitar los rendimientos estructurales de la citación bibliográfica: no sólo permite disponer de una forma de recuperación de información y, en concreto, de referencias bibliográficas relevantes en un determinado campo; además, la investigación de las referencias bibliográficas hace posible indagar vínculos disciplinares y redes intelectuales, y, adicionalmente, la medición de los índices de impacto introduce un cierto control de calidad de la investigación (De Bellis, 2009, p. xxi). 
En los estudios sociales de la ciencia, el interés por la citación se vincula a un énfasis en la construcción socio-histórica del conocimiento científico y en los estilos colectivos de inscripción y comunicación de la ciencia. Ya los precursores de un enfoque socio-histórico de la actividad científica remarcaron la importancia de los formatos de comunicación científica, de las modalidades de circulación de las publicaciones científicas, o de los movimientos persuasivos que hacen posible la consolidación de algún paradigma o tradición científica (Fleck, 1986; Kuhn, 1986; Feyerabend, 1981). Los estudios sociales sobre la ciencia y la tecnología se han centrado cada vez más en los aspectos retóricos de la construcción del conocimiento científico: las formas de inscripción, transformación y estilización de los enunciados, que permiten establecer hechos en la literatura científica; pero, también, los modos de comunicación estratégica de los hechos científicos, que involucran tanto una discusión persuasiva sobre la aceptabilidad, cuanto la aceptación por parte de los auditorios de la ciencia (Latour, 1992). En ese sentido, la citación desempeña una función retórica crucial en la construcción de los hechos científicos, ya que un enunciado deviene científico mediante la invocación estratégica de opiniones autorizadas, al apelar a abundantes referencias bibliográficas y explicitar los aliados en el texto; depende controversialmente de la reutilización de toda una red de artículos previos y de las publicaciones de usuarios posteriores (Latour, 1992, p. 31-49).

El énfasis en los aspectos retóricos de la construcción del conocimiento ha propiciado el surgimiento de una disciplina, la Retórica de la ciencia, que investiga el estilo de inscripción, los tópicos argumentativos y los movimientos persuasivos en el discurso científico. Este enfoque retórico ha conquistado un lugar propio frente a la filosofía de la ciencia y la historia de la ciencia, y su nicho específico de investigación se ha diferenciado del campo de la sociología de la ciencia (Harris, 1991). En el contexto de la Retórica de la ciencia, la citación ha concitado un indudable interés, ya que la referencia intertextual contribuyó decisivamente a la consolidación del sistema comunicativo de la ciencia moderna. La intertextualidad de la cita introduce un aspecto retórico decisivo en ese complejo entramado socio-discursivo de mediaciones entre autores, editores y públicos de la ciencia moderna: permite anclar los enunciados científicos en redes de literatura relevante y reestructurar persuasivamente las referencias disciplinares; de ese modo, informa estratégicamente las interacciones agonísticas entre pretensiones de validez, la crítica organizada, la 
discusión pública y el reconocimiento del prestigio científico, a través de una literatura científica cuya pieza fundamental será el artículo científico (Bazerman, 1988). En su reconstrucción histórica de las dimensiones retóricas del artículo científico, Gross, Harmon y Reidy (2002) dan cuenta de relevantes trasformaciones de los modos de presentación de la cita entre el siglo XVII y la actualidad, que apuntan cierta tendencia hacia el aumento de las citas y de la densidad citacional, a la uniformización de los sistemas de referencia, así como a la diferenciación precisa de la cita y de las referencias bibliográficas en el texto. Se trata de desarrollos que responden a la creciente importancia de los rendimientos argumentativos y de la comunicación de información en el artículo científico, en el contexto de comunidades científicas establecidas que recompensan la publicación de alto impacto y el aporte de datos funcionales para el sistema productivo. La presentación de las citas en el artículo científico contemporáneo responde a una creciente demanda de contextualización disciplinar y reconocimiento de crédito científico. De esa manera, los patrones de citación actual presentan una textura mucho más disciplinar y socializada (Gross, Harmon, Reidy, 2002).

La Lingüística aplicada y, particularmente, el análisis de los géneros del discurso científico, por parte de los estudios idiomáticos para propósitos académicos, aportan otro enfoque que se ha hecho cargo del análisis de la citación. Como parte del intento de clarificar el proceso de alfabetización académica desde una perspectiva multidimensional (cognitiva, social y lingüística), los estudios del lenguaje en contextos académicos han tratado de especificar las propiedades de los géneros discursivos, los presupuestos de la producción y comprensión de textos académicos, así como la adquisición de las competencias discursivas especializadas que hacen posible el desempeño experto en una disciplina (Bhatia, 2008; Parodi, 2010). Desde esta perspectiva de la Lingüística aplicada, la citación ha sido profusamente investigada para explicitar las formas de intertextualidad que atraviesan la escritura científica (Marinkovich \& Benítez, 2000), para explorar, a través de la referencia intertextual, las formas de incorporación de la voz del autor en el texto académico (Castelló et al., 2011), así como para reconocer las modalidades de posicionamiento metadiscursivo que, en el texto científico, modulan autorreflexivamente la interacción entre los autores, los textos y los lectores, de manera que se conforman comunidades discursivas y culturas disciplinares (Hyland $\&$ Tse, 2004). 
Ciertamente, el análisis de la intertextualidad ha generado una abundante literatura, en la que cabe reconocer ciertas diferencias de énfasis a la hora de comprender el elemento intertextual del discurso. El concepto de intertextualidad se asocia a cierto dialogismo de la enunciación, en virtud del cual todo enunciado discursivo aparece como respuesta activa a otros discursos, refiere y anticipa la palabra ajena, y recoge en sí polifónicamente numerosas voces enunciativas (Voloshinov, 1976). En otros casos, con el concepto de intertextualidad no se pretende remarcar el aspecto intersubjetivo de la enunciación; más bien, se cuestiona todo intento de contener, en una obra dependiente de un autor, la productividad ilimitada de las remisiones textuales. La intertextualidad designa entonces el cruce productivo de textos, la dispersión de lenguajes socio-históricos y la proliferación de citas textuales, que convierten a todo texto en una red multiforme de escrituras y múltiples citas culturales, sin que se pueda buscar una fuente originaria del sentido o un autor original (Kristeva, 1981, pp. 188-190; Barthes, 1994, p. 69-78). Existen usos más acotados del término que no asimilan la intertextualidad a cualquier tipo de remisión de un texto a otros. Genette (1989) califica como transtextuales a las formas más genéricas de presencia de un texto en otro, y reconoce diversas formas de transtextualidad, según el modo de inscripción de los textos ajenos. La intertextualidad es tan solo una de las modalidades de remisión transtextual, y concretamente consiste en una incorporación de enunciados, fragmentos o textos breves en un determinado marco textual (como ocurre en la cita, el plagio y la alusión). Por cierto, Genette diferencia la intertextualidad de la metatextualidad, ese tipo de remisión transtextual en que el texto ajeno suscita algún tipo de posicionamiento, comentario o relación crítica, sin que sea precisa la inscripción o incluso la referencia al texto comentado (Genette, 1989, p. 9-17). En ese sentido, cabe pensar que, al citar directa o indirectamente, se da una forma de remisión típicamente intertextual, en tanto que la referencia no integrada al enunciado (la mención del autor entre paréntesis al final de una frase o párrafo) es solo una atribución metatextual.

Como referencia intertextual, la cita exhibe cierta gramática inherente, ya que sus modalidades semióticas están sujetas a ciertas reglas de construcción y posibilidades estructurales. Desde el punto de vista de la gramática de la citación, cabe distinguir ciertas características de las cita, como su extensión (largas o cortas), su ubicación (al comienzo, en medio o al final del texto) o el tipo de marcadores lingüísticos (explícitos 
o implícitos) que las introducen (Plett, 1991, p. 8-9). En cuanto a la forma de inscripción gramatical de la cita, se pueden distinguir las citas en estilo directo o en estilo indirecto; pero, también, las citas integradas (que incorporan sintácticamente el nombre de la fuente en el propio texto citado) o las citas no integradas (en que la fuente aparece entre paréntesis al final del enunciado o en nota al pie, sin cumplir función sintáctica alguna) (Venegas, Meza \& Martínez, 2013). Las citas integradas pueden asumir distintas formas según las diferentes funciones sintácticas que desempeñe el nombre de la fuente citada: sujeto de la oración, complemento agente de una oración en voz pasiva, elemento de alguna cláusula oracional, o construcción adjunta introducida en la frase por un marcador discursivo del tipo "según" o "de acuerdo a" (Okamura, 2008). Cada una de estas formas de inscripción de la cita tiene rendimientos retóricos en la medida en que le da mayor o menor protagonismo a la voz del autor citado, y personaliza o despersonaliza los enunciados que se citan. En cada opción formal de realización textual están en juego distintas posibilidades de comprensión intertextual en relación con el estatuto aceptado o negociable del conocimiento, con el carácter controversial de las afirmaciones, con la participación dialógica en la discusión o con el compromiso del lector. Por ejemplo, las citas no integradas que se limitan a referir una fuente presentan el enunciado como conocimiento establecido que escapa a la controversia y no apela a la participación del lector; sin embargo, una cita integrada que sitúe la fuente en el sujeto oracional, e introduzca la cita con expresiones evaluativas, remarca el carácter negociable del conocimiento y el carácter controversial de las pretensiones, invita al diálogo y compromete al lector (Buckingham \& Nevile, 1997).

Los estudios del discurso citacional también han analizado la pragmática de la cita, es decir, las funciones que la cita desempeña en la interacción comunicativa. Las citas pueden confirmar y desarrollar orgánicamentelaargumentación deltexto; aveces, recogen planteamientos alternativos a los del texto; con frecuencia, señalan de modo superficial alguna fuente de información o referencia textual adicional; en ocasiones, introducen una relación negativa o crítica entre el pretexto y el texto que lo cita (Swales, 1986). Si atendemos a sus funciones argumentativas en el discurso científico, la citación puede aportar elementos persuasivos ligados a la pretensión del autor, a sus datos, a sus garantías, a sus fuentes 
y respaldos, o a ciertas reservas ante las tesis sostenidas (Angrosh, Cranefield \& Stanger, 2012). Las investigaciones empíricas sobre los usos de la citación en distintos niveles y géneros del discurso académico reconocen un amplio repertorio de funciones implicadas en las citas: las citas pueden atribuir una idea a algún otro autor para obtener autoridad intelectual, pueden ejemplificar argumentos, o referir algún antecedente para facilitar la búsqueda, pueden declarar el uso de herramientas investigativas de otros autores, o aplicar elementos teóricos de otras fuentes, pueden evaluar o comentar planteamientos ajenos, pueden relacionar fuentes, o realizar comparaciones y contrastes entre el propio enfoque y el de otros autores e, incluso, pueden demostrar competencia en el campo de investigación (Sánchez, 2013).

Cabría pensar que el amplio repertorio de formas y funciones de inscripción de la cita académica se ve afectado por la regulación disciplinar y la codificación de la citación en manuales de estilo. No obstante, la cita formalizada, altamente codificada y profesionalizada no deja de introducir efectos retóricos en el discurso científico: enmarca la experiencia de lectura (como un proceso lineal sembrado de referencias entre paréntesis, o como remisión a un paratexto ubicado a pie de página); aporta un medio de persuasión decisivo (mediante la autorización con fuentes canónicas o la indicación de evidencia reciente y novedosa), e incorpora a autores y lectores en comunidades discursivas de investigación (a través del ritual cortés de identificación con los pares, o mediante la exclusión de quienes no están al tanto de las referencias disciplinares obligadas) (Connors, 1999; Gilbert, 1977; Rose, 1996). Resulta pertinente, pues, preguntarse por el repertorio de formas y funciones de la citación en una ciencia social como la Psicología, que regula rígidamente la escritura académica bajo los dictados del manual de estilo de la APA, y, por otra parte, desde algunas de sus orientaciones nos invita a concebir el asunto de la Psicología de modo discursivo, como una construcción dialógica, relacional e, incluso, polifónica y marcada por una heterogeneidad de voces sociales y géneros discursivos (Shotter, 2001; Wertsch, 1991). ¿Cómo se maneja la inscripción de las diferentes voces discursivas en los textos del principal género del discurso académico de la Psicología, el artículo de investigación? ¿Qué tipo de intertextualidad se puede reconocer a través de las formas y funciones de la cita en el artículo de investigación de Psicología? 


\section{Método}

Este estudio se propone describir las formas de inscripción de las citas y las funciones argumentativas más frecuentes de la citación, en los artículos de investigación de dos revistas chilenas de Psicología publicados entre el 2008 y el 2012. Adicionalmente, se pretenden identificar los tipos de fuentes más citados en dichas publicaciones, para reconocer las principales influencias y las hegemonías intelectuales que enmarcan el discurso disciplinar de la Psicología chilena.

\subsection{Muestra}

El corpus textual analizado corresponde a la totalidad de los artículos de investigación publicados entre el 2008 y el 2012, en dos revistas chilenas de Psicología generalistas e indizadas: la Revista de Psicología de la Universidad de Chile y la revista Psykhe de la Universidad Católica de Chile. De un universo de 158 textos publicados en ambas revistas entre los años 2008 y 2012, se seleccionaron 106 artículos de investigación científica con la estructura recomendada por el manual de publicación de la disciplina (American Psychological Association, 2010) y se excluyeron textos como editoriales, ensayos, homenajes, reseñas o traducciones. Se escogen los artículos de estas dos revistas como muestra para la investigación por la cobertura temática generalista de ambas publicaciones, que permite obtener una perspectiva de conjunto de las líneas de la Psicología chilena (a diferencia de otras revistas especializadas en algún área disciplinar particular). Se considera la indización de ambas publicaciones, en la medida en que ello garantiza la selección y el reconocimiento de la calidad de los artículos publicados. La Revista de Psicología es la de más larga data en Chile y se encuentra indizada en la Red de Revistas Científicas de América Latina y el Caribe (Redalyc) y en Latindex, en tanto que la revista Psykhe está indizada en la Scientific Electronic Library Online (Scielo), en Redalyc y en Scopus. Se trata de publicaciones consolidadas, con más de veinte años de existencia, que están vinculadas a las primeras escuelas de Psicología que se fundaron en Chile. La distribución de artículos analizados por año se presenta en la tabla 1. 
Tabla 1

Artículos analizados por revista y año.

\begin{tabular}{lllllll}
\hline & 2008 & 2009 & 2010 & 2011 & 2012 & Total \\
\hline Revista de Psicología & 3 & 5 & 9 & 9 & 13 & 39 \\
\hline Psykhe & 15 & 15 & 8 & 16 & 13 & 67 \\
\hline Totales & 18 & 20 & 17 & 25 & 26 & 106 \\
\hline
\end{tabular}

\subsection{Procedimiento}

Para cumplir sus objetivos, este estudio realizó un análisis de contenido: se emplearon procedimientos sistemáticos y confiables al analizar los textos, para reconocer frecuencias de cierto tipo de elementos textuales (concretamente las formas y funciones de las citas, así como los tipos de fuentes más citadas), con el propósito de formular inferencias válidas sobre el contexto de producción y recepción de los textos analizados (Bardin, 1996; Krippendorf, 2004). Un detalle de las unidades de este análisis de contenido se representa en la tabla 2. La regla de análisis (Bardin, 1996) para todas las tareas de análisis contempladas consistió en la determinación de frecuencias. Para establecer las diferencias significativas entre las dos Revistas, se realizaron tablas de contingencias sujetas a la prueba estadística Chi cuadrado y al análisis de los residuos. 
Tabla 2

Unidades del análisis de contenidos de las fuentes, formas y funciones de la citación en los artículos de investigación de dos revistas de Psicología chilenas.

\begin{tabular}{|c|c|c|}
\hline $\begin{array}{l}\text { Objetivos } \\
\text { analíticos }\end{array}$ & $\begin{array}{l}\text { Unidad de } \\
\text { registro }\end{array}$ & Unidad de categorización \\
\hline $\begin{array}{l}\text { Describir las } \\
\text { formas de la } \\
\text { citación en los } \\
\text { artículos de } \\
\text { investigación } \\
\text { de la Psicología } \\
\text { chilena, entre el } \\
2008 \text { y el } 2012\end{array}$ & Cita & $\begin{array}{l}\text { Forma de la cita: } \\
\text { Directa integrada (reproducción } \\
\text { literal de menos de cuarenta palabras } \\
\text { y entrecomillada, con la fuente y la } \\
\text { página indicadas entre paréntesis al } \\
\text { final del enunciado). } \\
\text { Directa extensa (reproducción } \\
\text { literal de más de cuarenta palabras, } \\
\text { gráficamente separada en un párrafo } \\
\text { aparte, con la indicación de la fuente } \\
\text { y la página al final, entre paréntesis). } \\
\text { Indirecta (paráfrasis introducida } \\
\text { en una oración en estilo indirecto; } \\
\text { el nombre de la fuente cumple una } \\
\text { función sintáctica en la oración) } \\
\text { Referencial (indicación de fuentes } \\
\text { de algún enunciado, al final de } \\
\text { la oración y entre paréntesis; sin } \\
\text { integración al enunciado) }\end{array}$ \\
\hline
\end{tabular}

\begin{tabular}{|c|c|c|}
\hline $\begin{array}{l}\text { Describir las } \\
\text { funciones de } \\
\text { la citación en } \\
\text { los artículos de } \\
\text { investigación } \\
\text { de la Psicología } \\
\text { chilena actual }\end{array}$ & Cita & $\begin{array}{l}\text { Función de la cita: } \\
\text { Confirmatoria por cobertura } \\
\text { (la fuente citada autoriza las } \\
\text { pretensiones del artículo) } \\
\text { Confirmatoria por ejemplificación (la } \\
\text { fuente citada ilustra las afirmaciones } \\
\text { del artículo) } \\
\text { Crítica refutatoria (el artículo } \\
\text { polemiza con la fuente citada) } \\
\text { Crítica de reserva (la fuente citada } \\
\text { introduce una limitación a las } \\
\text { pretensiones del artículo) } \\
\text { Neutral alusiva (la fuente citada solo } \\
\text { añade información adicional) }\end{array}$ \\
\hline
\end{tabular}

Unidad de contexto

Secciones de la introducción, el marco teórico y la conclusión o discusión de cada artículo

Secciones de la introducción, el marco teórico y la conclusión o discusión de cada artículo

\begin{tabular}{|c|c|c|c|}
\hline $\begin{array}{l}\text { Identificar los } \\
\text { tipos de fuente } \\
\text { más citados en } \\
\text { los artículos de } \\
\text { investigación } \\
\text { de la Psicología } \\
\text { chilena actual }\end{array}$ & Cita & $\begin{array}{l}\text { Tipo de fuente: } \\
\text { Bibliográfica (disciplinar, no } \\
\text { disciplinar o compuesta) } \\
\text { Socio-gráfica (institucional o de } \\
\text { actores sociales) }\end{array}$ & $\begin{array}{l}\text { Secciones de la } \\
\text { introducción, } \\
\text { el marco } \\
\text { teórico y la } \\
\text { conclusión o } \\
\text { discusión de } \\
\text { cada artículo }\end{array}$ \\
\hline $\begin{array}{l}\text { Identificar las } \\
\text { fuentes más } \\
\text { referidas y su } \\
\text { procedencia } \\
\text { geográfica. }\end{array}$ & $\begin{array}{l}\text { Referencia } \\
\text { bibliográfica }\end{array}$ & $\begin{array}{l}\text { Nombres y apellidos de autores, } \\
\text { nombres de revistas y localización de } \\
\text { la plaza editorial. }\end{array}$ & $\begin{array}{l}\text { Sección de } \\
\text { referencias } \\
\text { bibliográficas } \\
\text { de cada } \\
\text { artículo }\end{array}$ \\
\hline
\end{tabular}




\section{Resultados}

Se categorizaron un total de 3579 citas, distribuidas entre las dos revistas como se indica en la tabla 3. Como se puede apreciar, el promedio de citas por artículo es levemente mayor en la revista Psykhe.

Tabla 3

Distribución de las citas categorizadas por revista y promedio de citas por artículo.

\begin{tabular}{lccc}
\hline & Total de citas & $\begin{array}{l}\text { Número de } \\
\text { artículos }\end{array}$ & $\begin{array}{l}\text { Promedio de citas } \\
\text { por artículo }\end{array}$ \\
\hline Revista de Psicología & 1170 & 38 & 30,8 \\
\hline Psykhe & 2409 & 68 & 35,4 \\
\hline Totales & 18 & 20 & 17 \\
\hline
\end{tabular}

\subsection{Forma de inscripción de las citas}

En las citas de los artículos de investigación analizados se aprecia una mayor frecuencia de las citas referenciales, por sobre las citas indirectas o directas, como se observa en la tabla 4. Además, existen diferencias significativas en la forma de citación de las dos revistas analizadas: la revista Psykhe emplea más citas referenciales que la Revista de Psicología, mientras que esta última utiliza más citas directas que la primera.

Tabla 4

Frecuencia de las distintas formas de inscripción de la cita en los artículos analizados.

\begin{tabular}{lcccc}
\hline & $\begin{array}{l}\text { Cita } \\
\text { referencial }\end{array}$ & Cita indirecta & $\begin{array}{l}\text { Cita directa } \\
\text { integrada }\end{array}$ & $\begin{array}{l}\text { Cita directa } \\
\text { extensa }\end{array}$ \\
\hline Revista de Psicología & $66,3 \%$ & $26,8 \%$ & $4,9 \%$ & $2,0 \%$ \\
\hline Psykhe & $72 \%$ & $25,5 \%$ & $2,1 \%$ & $0,4 \%$ \\
\hline Totales & 18 & 20 & 17 & \\
\hline$\chi^{2}=46,32 ; \mathrm{p}=4.826 \mathrm{e}-10$ & &
\end{tabular}




\subsection{Función de la citación}

Se reconoce un predominio de las citas confirmatorias que sostienen las pretensiones argumentativas del autor, y particularmente destaca el tipo de cita que introduce algún enunciado o fuente para suministrar cobertura, como se aprecia en la tabla 5. Las citas confirmatorias son más frecuentes que las citas neutrales alusivas o que las citas críticas, como se aprecia en el gráfico 1. Existen leves diferencias entre las dos revistas, en lo que concierne al empleo de la cita crítica, que es mayor en la Revista de Psicología, como se aprecia en la tabla 6.

\section{Tabla 5}

Frecuencia de las funciones de las citas en los artículos analizados.

\begin{tabular}{lccccc}
\hline & $\begin{array}{l}\text { citas } \\
\text { confirmatorias } \\
\text { por cobertura }\end{array}$ & $\begin{array}{l}\text { cita neutral } \\
\text { alusiva }\end{array}$ & $\begin{array}{l}\text { citas } \\
\text { confirmatorias } \\
\text { por } \\
\text { ejemplificación }\end{array}$ & $\begin{array}{l}\text { citas críticas } \\
\text { refutatorias }\end{array}$ & $\begin{array}{l}\text { citas críticas } \\
\text { de reserva }\end{array}$ \\
\hline $\begin{array}{l}\text { Revista de } \\
\text { Psicología }\end{array}$ & $58,7 \%$ & $26,3 \%$ & $6,7 \%$ & $4,4 \%$ & $3,8 \%$ \\
\hline Psykhe & $59,3 \%$ & $25,5 \%$ & $9,2 \%$ & $3,4 \%$ & $2,6 \%$ \\
\hline Totales & $59,12 \%$ & $25,78 \%$ & $8,35 \%$ & $3,74 \%$ & $2,98 \%$ \\
\hline
\end{tabular}

$\chi^{2}=12.7098 ; \mathrm{p}=0.01278$

Tabla 6

Frecuencias de las citas confirmatorias, neutrales alusivas y críticas.

\begin{tabular}{llll}
\hline & citas confirmatorias & $\begin{array}{l}\text { citas neutrales } \\
\text { alusivas }\end{array}$ & citas críticas \\
\hline Revista de Psicología & $65,40 \%$ & $26,30 \%$ & $8,30 \%$ \\
\hline Psykhe & $68,50 \%$ & $25,50 \%$ & $6,00 \%$ \\
\hline Totales & 18 & 20 & 17 \\
\hline$\chi^{2}=7.58 ; \mathrm{p}=0.02262$ & &
\end{tabular}




\subsection{Tipos de fuente más citados}

Por lo que respecta a si las citas provienen de fuentes bibliográficas, discursos sociales o institucionales, se aprecia un predominio de las fuentes bibliográficas disciplinares en los totales de ambas revistas, como se observa en las tabla 7. Existen algunas diferencias significativas entre ambas revistas: la Revista de Psicología presenta un mayor número de citas bibliográficas no disciplinares, mientras en la revista Psykhe habría mayor número de citas bibliográficas disciplinares; además, en la Revista de Psicología hay comparativamente mayor número de citas sociográficas.

Tabla 7

Frecuencia de los distintos tipos de fuente citados en los artículos analizados.

\begin{tabular}{lcclll}
\hline & $\begin{array}{l}\text { citas } \\
\text { bibliográficas } \\
\text { disciplinares }\end{array}$ & $\begin{array}{l}\text { citas } \\
\text { bibliográficas } \\
\text { no } \\
\text { disciplinares }\end{array}$ & $\begin{array}{l}\text { citas } \\
\text { bibliográficas } \\
\text { compuestas } \\
\text { (disciplinares } \\
\text { y no } \\
\text { disciplinares) }\end{array}$ & $\begin{array}{l}\text { citas } \\
\text { sociográficas } \\
\text { de fuentes } \\
\text { institucionales }\end{array}$ & $\begin{array}{l}\text { citas } \\
\text { sociográficas } \\
\text { de actores } \\
\text { sociales }\end{array}$ \\
\hline $\begin{array}{l}\text { Revista de } \\
\text { Psicología }\end{array}$ & $40,2 \%$ & $44,4 \%$ & $7,9 \%$ & $6,6 \%$ & $0,9 \%$ \\
\hline Psykhe & $56,3 \%$ & $30,2 \%$ & $8,6 \%$ & $4,8 \%$ & $0,0 \%$ \\
\hline Totales & $51,01 \%$ & $34,82 \%$ & $8,41 \%$ & $5,39 \%$ & $0,33 \%$ \\
\hline \multicolumn{2}{c}{$\chi^{2}=109.24 ; \mathrm{p}<2.2 \mathrm{e}-16$} & & & \\
\hline
\end{tabular}


Si agrupamos los distintos tipos de citas bibliográficas y, por otra parte, las citas que recogen voces de actores sociales, personales o institucionales, se pone de manifiesto un claro predominio de las citas bibliográficas, que refieren otros textos académicos.

\subsection{Fuentes más referidas y hegemonías geográficas}

Como puede apreciarse en la tabla 8 , la lista de autores más referidos en las bibliografías de los artículos está encabezada por Michel Foucault (un autor en los márgenes de la Psicología y con un discurso crítico difícilmente encasillable en la disciplina, al igual que Judith Butler, que figura en segundo lugar). En el primer lugar de la lista de revistas más citadas figura una de las revistas chilenas analizadas, la revista Psykhe; pero, entre las seis revistas más citadas, encontramos cuatro publicaciones internacionales de la corriente principal con un alto índice de impacto.

Tabla 8

Los seis autores y las seis revistas más frecuentemente referidos en la bibliografía de los artículos analizados.

\begin{tabular}{lclc}
\hline Autores & Referencias & Revistas & Referencias \\
\hline Foucault, Michel (Francia) & 21 & Psykhe & 79 \\
\hline $\begin{array}{l}\text { Butler, Judith (Estados } \\
\text { Unidos) }\end{array}$ & 15 & Child Development & 42 \\
\hline $\begin{array}{l}\text { Vinet, Eugenia (Chile) } \\
\text { Strauss, Anselm (Estados }\end{array}$ & 15 & $\begin{array}{l}\text { Journal of Personality and } \\
\text { Social Psychology }\end{array}$ & 38 \\
\hline $\begin{array}{l}\text { Unidos) } \\
\text { Montero, Maritza }\end{array}$ & 14 & Developmental Psychology & 22 \\
\hline Venezuela) & 14 & $\begin{array}{l}\text { Journal of Educational } \\
\text { Psychology }\end{array}$ & 22 \\
\hline \begin{tabular}{l} 
Dubar, Claude (Francia) \\
\hline
\end{tabular}
\end{tabular}

Respecto a la procedencia geográfica de las fuentes referidas en la bibliografía del total de los artículos analizados, predominan las plazas editorialeslatinoamericanas por sobre las norteamericanas o europeas, como se observa en las tablas 9 y 10. Si agrupamos las plazas norteamericanas y europeas, puede apreciarse una marcada influencia de la literatura proveniente de esos continentes y, por ende, de la corriente principal. La revista Psykhe presenta un mayor porcentaje de referencias bibliográficas de plazas editoriales norteamericanas, que la Revista de Psicología; esta última presenta más referencias latinoamericanas que la Revista Psykhe. 
Tabla 9

Las seis plazas editoriales más referidas en la bibliografía de los artículos analizados.

\begin{tabular}{lcccccc}
\hline & $\begin{array}{c}\text { Estados } \\
\text { Unidos }\end{array}$ & Chile & España & Inglaterra & Argentina & México \\
\hline $\begin{array}{l}\text { Revista de } \\
\text { Psicología }\end{array}$ & 209 & 221 & 214 & 65 & 124 & 61 \\
\hline Psykhe & 576 & 496 & 228 & 145 & 81 & 72 \\
\hline Total & 785 & 717 & 442 & 210 & 205 & 133 \\
\hline
\end{tabular}

Tabla 10

Procedencia geográfica de las plazas editoriales más referidas en las Bibliografías de los artículos analizados.

\begin{tabular}{lcccc}
\hline & Latinoamérica & Norteamérica & Europa & $\begin{array}{c}\text { África, Asia, } \\
\text { Oceanía }\end{array}$ \\
\hline Revista de Psicología & $49,9 \%$ & $21,3 \%$ & $28,5 \%$ & $0,4 \%$ \\
\hline Psykhe & $38,6 \%$ & $33,2 \%$ & $27,7 \%$ & $0,4 \%$ \\
\hline Total & $42,68 \%$ & $28,9 \%$ & $28,01 \%$ & $0,4 \%$ \\
\hline
\end{tabular}

$\chi^{2}=51.116 ; \mathrm{p}=4.62 \mathrm{e}-11$

\section{Discusión}

¿Qué tipo de intertextualidad se esboza a través del formato de citación de los artículos de Piscología analizados? Sin duda, la forma de inscripción del discurso ajeno en las citas de los artículos de Psicología analizados no parece propiciar particularmente que se dé cuenta de la condición polifónica de los discursos sociales (como pretenden algunas orientaciones discursivas que han ganado terreno en la teoría y las metodologías de la Psicología contemporánea). En la medida en que predomina una forma de inscripción de la cita meramente referencial o en estilo indirecto, se borra la textura de la voz citada y se pierde el tono propio de cada discurso convocado. La textura singular de la voz del otro no resuena demasiado cuando se da un formato de cita referencial, que solo indica posibles fuentes, pero no inscribe directamente la palabra 
ajena ni integra su perspectiva en la propia enunciación. En ese sentido, cabe pensar que el predominio de la cita referencial pone de manifiesto un escaso compromiso con la palabra ajena; no en vano, este tipo de cita indirecta y no integrada parece centrar el foco en la idea, más que en las voces discursivas (Venegas, Meza \& Martínez, 2013), y produce un cierto distanciamiento retórico y epistémico que, tal vez, no puede separarse del afán de justificación autorizadora. Por otra parte, las funciones de la citación más frecuentes en los artículos de Psicología analizados consisten en la confirmación de las pretensiones del autor o en la mención neutral alusiva, y no se suscitan las formas de respuesta activa que son propias del uso crítico de la citación. En ese sentido, hay cierta concordancia entre la forma referencial y cierta función atributiva que remite a las ideas y sus autores, para así capitalizar la autoridad de las fuentes y demostrar dominio del campo disciplinar (Sánchez, 2013).

Los tipos de fuentes que más se repiten en las citas son las referencias bibliográficas (sobre todo, las citas disciplinares provenientes de la corriente principal de la Psicología norteamericana y europea), de manera que los artículos de Psicología analizados se ven privados de la presencia de la voz de los actores sociales locales que supuestamente son el asunto de la disciplina. Este formato hegemónico de citación (la cita referencial confirmatoria o neutralmente alusiva de fuentes bibliográficas disciplinares) concreta una retórica determinada en que las pretensiones discursivas de validez aparecen como algo fundado en conocimiento establecido; se trata de un discurso disciplinar autorreferencial no negociable ni susceptible de controversia, que no invita particularmente al diálogo comprometido del lector. Semejante práctica citacional de los artículos de Psicología analizados contribuye así a una clausura del discurso disciplinar experto, por medio de la referencia intertextual a toda una red de fuentes autorizadoras y consagradas en la corriente principal; además, la remisión intertextual resulta capitalizada mediante toda una economía de la citación y de la maximización del impacto bibliométrico.

Cabe pensar que las diferencias observadas entre las prácticas citacionales de las dos revistas analizadas no se deben únicamente a la diferencia de sus líneas editoriales (la Revista de Psicología, más comprometida con los tópicos de las subjetividades y los asuntos sociales; la revista Psykhe, más enfocada en la investigación estándar de corte empírico) o a las limitaciones en la extensión del texto de 
los artículos (ocho mil palabras), que inducen a referir el estado del arte en vez de citar. Tal vez habría que considerar la diferencia en indización de ambas revistas para dar cuenta de la prevalencia de citas referenciales disciplinares en la revista Psykhe: la indización de una publicación involucra el cumplimiento de normas de escritura altamente estandarizadas, como las normas APA, que regulan exhaustivamente los formatos de citación; además, el registro del índice de impacto de los artículos estimula la capitalización de la cita disciplinar referente al estado del arte.

Ulteriores investigaciones sobre las prácticas citacionales en los distintos géneros discursivos de diferentes ciencias permitirán discutir hasta qué punto se extienden la retórica y el ethos argumentativo asociados a la citación en los artículos analizados. ¿Se trata de algo exclusivo del género del artículo de investigación de una ciencia social como la Psicología, particularmente tensionada por la necesidad de presentarse como una ciencia estándar, dotada de un nicho académico y profesional propio, con redes y normas de publicación expertas?

\section{Conclusión}

El tipo de intertextualidad que cabe reconocer en las citas de los artículos de investigación de las revistas analizadas no corresponde precisamente a cierto dialogismo: los enunciados no propician la respuesta activa a otros discursos ni recogen polifónicamente otras voces socio-históricas. Tampoco se trata del tipo de intertextualidad disruptiva que abre el texto a una dispersión productiva de escrituras entrecruzadas y de citas heterogéneas. En fin, las prácticas de la citación encontradas en los artículos analizados se alejan de una intertextualidad abierta, y están más cerca de cierta metatextualidad, esa forma de remisión transtextual en que el texto ajeno se inscribe indirectamente a través de algún posicionamiento, comentario o relación crítica (Genette, 1989, p. 13). La cita en los artículos de Psicología analizados opera básicamente al servicio del posicionamiento metadiscursivo, que regula la relación entre autor, texto y lector, y permite conformar una comunidad discursiva experta y una cultura disciplinar autorreferente (Hyland \& Tse, 2004).

Por otra parte, la retórica de la citación en los artículos de Psicología analizados tiene rendimientos específicamente epistemológicos, que cuestionan el ethos atribuido a la actividad científica: el universalismo 
científico, ligado a criterios cognoscitivos impersonales y preestablecidos; el carácter cooperativo de la investigación científica y su contribución constante a la comunidad; cierto desinterés cognoscitivo sujeto al control público, y el escepticismo organizado que la ciencia exhibe como autocrítica falibilista (Merton, 1964, p. 542-552). Las estrategias retóricas asociadas a la citación en los artículos de Psicología analizados ponen de manifiesto un ethos algo distinto: el "glocalismo" de las redes de investigación, que afirma vínculos comunitarios locales entre investigadores, a través de referencias disciplinares globales de la corriente principal; la autorreferencia del discurso profesional y experto; la capitalización simbólica de la citación en toda una economía que persigue la maximización del impacto bibliográfico y el atesoramiento de crédito; el discurso autoconfirmatorio y la referencia meramente alusiva al estado del arte, en desmedro de los posicionamientos críticos. En suma, la condición intertextual (o, más bien, metatextual) de la citación en los artículos de Psicología tiene consecuencias retóricas y epistemológicas decisivas en la construcción discursiva del conocimiento disciplinar.

\section{Referencias bibliográficas}

American Psychological Association. (2010). Publication Manual of the American Psychological Association. (6a ed.). Washington, DC: American Psychological Association.

Angrosh, M. A., Cranefield, S., \& Stanger, N. (2012). A Citation Centric Annotation Scheme for Scientific Articles. Proceedings of Australasian Language Technology Association Workshop, 5-14.

Bardin, L. (1996). Análisis de contenido. Madrid: Ediciones Akal.

Barthes, R. (1994). El susurro del lenguaje. Barcelona: Paidós.

Bazerman, C. (1988). Shaping Written Knowledge: The Genre and Activity of the Experimental Article in Science. Madison, Wisconsin: University of Wisconsin Press.

Bhatia, V. (2008). Lenguas con Propósitos Específicos: Perspectivas cambiantes y nuevos desafíos. Revista Signos, 41(67), 157-176. 
Buckingham, J. \& Nevile, M. (1997). A Model of Citation Options. Australian Review of Applied Linguistics, 20(2), 5166.

Castelló, M., Corcelles, M., Iñesta, A., Bañales, G. \& Vega, N. (2011). La voz del autor en la escritura académica: Una propuesta para su análisis. Revista Signos, 44(76) 105-117.

Connors, R. J. (1999). The Rhetoric of Citation Systems, Part II: Competing Epistemic Values in Citation. Rhetoric Review, 17(2), 219-245.

Cronin, B. (1998). Metatheorizing Citation. Scientometrics, 43(1), 45-55.

De Bellis, N. (2009). Bibliometrics and Citation Analysis: from the Science Citation Index to Cybermetrics. Lanham, Maryland: Scarecrow Press.

Feyerabend, P. (1981). Tratado contra el método. Madrid: Tecnos.

Fleck, L. (1986). La génesis y el desarrollo de un hecho cientifico. Madrid: Alianza Editorial.

Garfield, E. (2009). The Evolution of the Science Citation Index. Contributions to Science, 5(1), 63-70.

Genette, G. (1989). Palimpsestos. Madrid: Taurus.

Gilbert, G. N. (1977). Referencing as Persuasion. Social Studies of Science, 7, 113-122.

Gross, A. G., Harmon, J. E. \& Reidy, M. (2002). Communicating Science: The Scientific Article from the 17th Century to the Present. New York: Oxford University Press.

Harris, R. A. (1991). Rhetoric of Science. College English, 53(3), 282-307.

Hyland, K. \& Tse, P. (2004). Metadiscourse in Academic Writng: A Reappraisal. Applied Linguistics, 25(2), 156-177.

Krippendorf, K. (2004). Content analysis: An introduction to its methodology. California: Sage Publications.

Kristeva, J. (1981). Semiótica I. Madrid: Fundamentos. 
Kuhn, T. S. (1986). La estructura de las revoluciones científicas. Madrid: Fondo de Cultura Económica.

Latour, B. (1992). Ciencia en acción. Barcelona: Editorial Labor.

Marinkovich, J. \& Benítez, R. (2000). Aproximaciones al análisis intertextual del discurso científico. Revista Signos, 33(48), 117-128.

Merton, R. (1964). Teoría y estructura sociales. México, D. F.: Fondo de Cultura Económica.

Okamura, A. (2008). Citation Forms in Scientific Texts: Similarities and Differences in L1 and L2 Professional Writing. Nordic Journal of English Studies, 7(3), 61-81.

Parodi, G. (Ed.). (2010). Alfabetización académica y profesional en el siglo XXI: Leer y escribir desde las disciplinas. Santiago de Chile: Ariel.

Plett, H. (Ed.). (1991). Intertextuality. New York: de Gruyter.

Rose, S. K. (1996). What's Love Got to Do with It? Scholarly Citation Practices as Courtship Rituals. Journal of Language and Learning across the Disciplines, 1(3), 36-47.

Sánchez, D. (2013). Aplicabilidad de la tipología de funciones retóricas de las citas al género de la memoria de máster en un contexto transcultural de enseñanza universitaria. Revista Signos, 46(81), 82-104.

Shotter, J. (2001). Realidades conversacionales. Amorrortu: Buenos Aires.

Swales, J. (1986). Citation Analysis and Discourse Analysis. Applied Linguistics, 7, 39-56.

Venegas, R., Meza Guzmán, P. \& Martínez Hincapié, J. (2013). Procedimientos discursivos en la atribución del conocimiento en tesis de lingüística y filosofía en dos niveles académicos. Revista de Lingüística Teórica y Aplicada, 51(1), 153-179.

Wertsch, J. V. (1991). Voces de la Mente. Madrid: Visor.

White, H. D. (2004). Citation Analysis and Discourse Analysis Revisited. Applied Linguistics, 25(1), 89-116. 